\title{
The Effect of Model Fidelity on Real-Time Optimization Performance
}

\author{
W. S. Yip and T. E. Marlin \\ Department of Chemical Engineering, McMaster University, Hamilton, ON L8S 4L7, Canada
}

\begin{abstract}
The ability of a real-time optimization (RTO) system to track the changing optimum closely relies on an accurate model for representing the plant behavior. This paper investigates the effect of model fidelity on RTO performance using a simulated industrial boiler network case study. Three optimization approaches with very different modelling fidelity are investigated; 1) model-free direct search method, 2) modelbased method using a simplified efficiency curve model, and 3) model-based method using a fundamental model. The model-free direct search method builds a locally linear model using empirical data. It takes many steps to reach the optimum, which causes a significant profit loss during tracking. This tracking loss can be reduced by using the model-based RTO system. The RTO system with an updated, detailed fundamental model is able to track fast and large disturbances because the model is accurate in a large range of operation. An RTO system with a simplified efficiency model requires periodic experimentation to correct for the disturbances, which can cause significant profit loss during experimentation and tracking. This study demonstrates how quantitative performance measures improve as higher fidelity models are used in real-time operations optimization.
\end{abstract}

\section{Introduction}

Real-time operations optimization (RTO) of steady-state chemical plant has become attractive in improving the operating profit (Culter \& Perry, 1983; Darby \& White, 1988; Marlin \& Hrymak, 1997). There are two typical approaches in RTO: 1) model-free direct search methods and 2) model-based methods. Model-free direct search methods track the optimum based on the gradient of plant performance estimated from the plant data. Model-based methods make use of a fundamental model to represent the plant, and the optimum is determined by optimizing the plant performance subject to the model. The RTO performance depends on the accuracy of the plant model. This paper investigates the crucial factors in deciding the model structure to be used in RTO systems.

A typical model-based closed-loop RTO system shown in Figure 1 includes the following subsystems: 1) updating of model parameters using plant measurements, 2) optimization of the updated model, and 3) process control. Measurements are taken from the plant, validated and low-pass filtered. Process parameters are estimated from the measurements in the model updater to minimize the mismatch between the model prediction and plant measurements. The optimum operating policy is determined by optimization of the updated model. The optimizer results are implemented by process controllers.

An RTO system relies on model updating as feedback to correct for modelling errors and disturbances and to enable the RTO system to track the optimum closely. Therefore, good RTO performance, i.e. a small offset between the true plant optimum and noise-free model prediction and small variability of the prediction (Forbes and Marlin, 1996), can be achieved by using a high fidelity model for optimization and having data sets with sufficient information content for accurate parameter estimation. Plant variation in the data sets is required to have sufficient information content to reliably estimate the adjustable parameters, which can be generated by disturbances (Yip and Marlin, 2001a) and designed experiment (Yip and Marlin, 2001b). This paper compares the RTO performance achieved using three modelling/optimization approaches for boiler load optimization. These are; 1) model-free direct search methods, 2) model-based methods using a simplified efficiency curve model, and 3) model-based methods using a fundamental model. This case study can provide guidelines on selecting the appropriate optimization approach for RTO application in a boiler network system. 


\section{Description of the Boiler Network Case Study}

A boiler network is selected to investigate the effect of model fidelity on RTO performance because it is commonly used in chemical plants for generating steam for heat and power. Typically, a number of boilers supply steam to a common header to satisfy the steam demand, and these boilers have different performance or efficiency. Opportunity exists for optimization to distribute the steam demand to different boilers to maximize the overall boiler network efficiency.

The boilers used in this paper are modified from the boiler in a steel mill. Details of the configuration of the boiler and the fundamental model for the plant are reported in Yip (2002). Superheated steam is produced by heating water/steam in generating tubes, risers and superheaters using the hot flue gas generated by combustion of fuel with air. The dominant mode of heat transfer in generating tubes and superheaters is convection, while the dominant mode of heat transfer in risers is radiation. Part of the heat in the flue gas is recovered by heating the feed water and incoming air in the economizer and air preheaters, respectively.

Boiler performance is measured by boiler efficiency which is defined as the ratio of the energy absorbed by the feed water to generate superheated steam and energy input to the boiler from combustion of fuel. Different boilers have different efficiency curves as a function of steam load, depending on the boiler design and operating conditions such as fuel composition, blowdown flow and air/fuel ratio. In this paper, three boilers with different heat transfer areas and hence different efficiency curves are used. The boiler design and operating conditions are reported in Yip (2002), and the efficiency curves are shown in Figure 2.

The shape of the efficiency curve has a maximum when the steam load is varied. At high boiler load, more energy is required to produce superheated steam. Although the high flue gas velocity increases the heat transfer coefficient, the flue gas temperature exiting the boiler increases. This increased loss to the environment results in a lower efficiency. At low boiler capacity, excess air has to be increased to generate turbulent mixing of fuel and air (Dukelow, 1991). Part of the heat from burning fuel is wasted in heating the excess air, so that the efficiency drops at low steam load.

\section{Strategies of the Closed-Loop Optimization of Boiler Network}

Three approaches are implemented to optimize the boiler network in real time. The objective is to maximize the overall efficiency of the boiler network for the disturbances in demand, fuel composition and heat exchanger fouling. The boiler network consists of three boilers supplying steam to a common header. Therefore, the optimization variables are the steam loads of two of the boilers because the steam demand must be satisfied. In the direct search methods, the optimum is determined solely from the plant data without using a rigorous model. In the model-based approaches, the RTO system shown in Figure 1 is used for tracking the changing optimum. Two different modelling alternatives are used in the modelbased methods, the simplified efficiency curve model and fundamental model. The following subsections discuss briefly these optimization approaches.

\subsection{Direct search method}

A direct search method tracks the changing optimum by using the local derivative of the plant performance estimated from the plant data without using a rigorous mathematical model. The method presented by Bozenhardt (1986) is used in this paper. The plant performance is assumed to be a linear function of the optimization variables as shown in (1)

$$
\mathrm{P}(\mathbf{x})=\mathrm{P}_{0}+\mathbf{g}^{\mathrm{T}} \mathbf{x}
$$

where $\mathrm{P}$ is the plant performance (overall boiler network efficiency), and $\mathbf{x}$ is the vector of the optimization variables (two boiler loads, because the total steam produced must meet the demand). $\mathrm{P}_{0}$ and the elements in the vector $\mathbf{g}$ are the model parameters that are estimated from the plant data. These parameters are estimated by the least squares method using several recent data sets. The direction of the 
vector $\mathbf{g}$ is the direction of improving the plant performance. The plant is moved along that direction with a fixed step size, which is a tuning parameter in this method. After the plant movement, a new data set is available, the oldest data set is discarded, and the above calculation is repeated. In this case study, five data sets are used for parameter estimation and a step size of $0.1 \mathrm{klb} / \mathrm{h}$ is used for the optimization move.

\subsection{Model-based method using the simplified efficiency curve model}

In the simplified efficiency curve model, a second order polynomial in (2) (Green \& ai-Shaikh, 1980) is used for approximating the efficiency curves shown in Figure 2 for each boiler

$$
\eta_{\mathrm{i}}=\mathrm{A}_{\mathrm{i}}+\mathrm{B}_{\mathrm{i}} \mathrm{F}_{\mathrm{i}}+\mathrm{C}_{\mathrm{i}} \mathrm{F}_{\mathrm{i}}^{2}
$$

where $\eta$ is the boiler efficiency, F is the steam load, A, B and C are the updated parameters, and i denotes the boiler number. The overall boiler network efficiency is maximized subject to the model in (2) and the material balance equation in the steam header in which the sum of the steam loads is equal to the steam demand.

Updating the parameters in the second order polynomial in the efficiency curve model in (2) requires multiple (historical) data sets at different steam loads. Data sets with different steam loads can be generated by disturbances in demand or periodic experimentation. The disturbances in fuel composition and heat exchanger fouling can change the shape of the boiler efficiency curve. Since the shape of the efficiency curve for each boiler is required for optimization, experiments are needed to generate new data sets for updating the parameters in (2).

The expected profit experimental design criterion (Yip and Marlin, 2001b) is implemented to design plant experiments for updating the efficiency curve model. This experimental design criterion considers profit explicitly in designing plant experiments for RTO systems to find the best tradeoff between the profit gain due to improved modelling and the profit loss before and during experimentation. The objective function for the experimental design, which is the total expected profit over a future horizon after an unmeasured disturbance has occurred, consists of three terms. The first term is the profit change due to offset, the second term is the profit loss due to experimentation, and the third term is the profit change due to variability. The profit change due to offset is estimated based on the anticipated size and speed of disturbance from process knowledge. The loss in profit during experimentation is estimated from the current model optimum and its reduced Hessian matrix. Therefore, low cost experiments are close to the current optimum or along the ridge of the profit contours to reduce this loss. To increase the profit gain due to an improved model, large plant perturbations are beneficial to improve the information content in the data sets. By maximizing the total expected profit, the time to start experimentation and the experimental operating conditions are designed to trade off the profit loss before and during experimentation and the profit gains after experimentation by reducing the offset and variability.

The model using an updated efficiency curve is optimized subject to a "trust region" in which the approximated model is locally accurate. The trust region is required because the model is valid within the operating or data window bounded by the historical data sets. However, the trust region is defined to be larger than the data window for extrapolation, because if the model is optimized subject to the data window in which the model is valid, the optimum cannot be tracked. The model is assumed to be accurate over a small region extended from the region defined by the data window. The size of the trust region is the tuning parameter in this strategy. When the model optimum is reached, the data window will bracket the optimum.

\subsection{Model-based method using the fundamental model}

A detailed fundamental model could be accurate over a wider range of operation and might be able to track the changing optimum even for large disturbances. In this case study, the fundamental model described in Yip (2002) is used in the RTO system. Each heat exchanger is modelled by material and energy balance equations, pressure drop equation for fluid flow and log mean temperature difference model for heat transfer. Thermodynamic and transport properties are derived by fitting the physical data 
with temperature and pressure using polynomials. Combustion of fuel with air in the furnace is assumed to be complete, and a uniform gas temperature is assumed in the furnace.

In the fundamental model, the updated parameters have physical meanings, such as the heat transfer coefficients and heating value of fuel. Updating the heat transfer coefficients in the heat exchangers require temperature measurements of the fluids entering and leaving the heat exchangers. A single data set is used for updating, because experience shows that experimentation is not required for updating in this boiler network case study.

\subsection{Measurements for the closed-loop optimization}

Different optimization approaches require different sets of measurements. In the direct search and simplified efficiency curve model approaches, the fuel heating value measurement is required to estimate the boiler efficiency using the input-loss method (Dukelow, 1991; Payne, 1985). When using a detailed fundamental model, fuel heating value measurement is not required, because the fuel heating value can be estimated from the available flow and temperature measurements. The lists of measurements used for updating in different optimization approaches are reported in Yip (2002).

\subsection{Model Mismatch}

The model representing the plant in the closed-loop RTO studies was different from any of the models used in the RTO optimizers. Naturally, the "fundamental" RTO model was closest to the plant, but mismatch in structure and parameters existed, even with the most rigorous RTO approach.

\section{Case Study Results}

Case studies are designed to investigate the RTO performance of different optimization approaches for tracking commonly encountered disturbances in a boiler network. The disturbances considered in this case study are the fast and slow periodic changes in steam demand and fuel composition and continuous fouling of heat exchangers.

The RTO performances are compared based on the overall boiler network efficiency attained and the magnitudes of the change in steam loads, $\Delta \mathrm{F}$. The magnitude of the change in steam loads is expressed as the sum of the change in steam loads between successive RTO executions for all the boilers as stated in (3)

$$
\|\Delta \mathrm{F}\|=\left(\sum_{\mathrm{i}=1}^{\mathrm{N}_{\text {bilier }}}\left(\mathrm{F}_{\mathrm{i}}(\mathrm{N})-\mathrm{F}_{\mathrm{i}}(\mathrm{N}-1)\right)^{2}\right)^{\frac{1}{2}}
$$

where $\mathrm{N}$ is the RTO execution, and $\mathrm{N}_{\text {boiler }}$ is the number of boilers which is equal to 3 in this case study.

Typical performance measured by the efficiency attained for a periodic disturbance change in fuel composition is shown in Figure 3. The dotted line is the optimum efficiency calculated by using a perfect plant model with exact measure of disturbances. This optimum efficiency is not realistically achievable, but it is a basis for comparing performance. The RTO system using the fundamental model can track the changing optimum closely. The fundamental model, which is accurate over a wide range of operation, is able to predict the boiler performance even if there is a large disturbance in fuel composition. It takes a few steps to reach the optimum after the disturbance change, which is especially advantageous for high disturbance frequency. The RTO system using the simplified efficiency curve model with experimentation requires more steps to reach the optimum. During experimentation, there is a loss in boiler network efficiency. The optimum may not be reached immediately after experimentation because the trust region defined from the data window restricts the plant movement. The efficiency loss during experimentation and tracking can be significant for fast disturbances. The direct search method is unable to track the optimum closely because the linear model in (1) is accurate locally. A small step size is chosen to restrict the plant movements to avoid excessive extrapolation of the linear model. Therefore, the 
direct search method requires many steps to reach the optimum and is not suitable for tracking fast disturbances.

The distribution of the magnitudes of change in steam loads is shown in Figure 4. There are significantly larger magnitudes of change in steam loads $\left(\|\Delta \mathrm{F}\|>1 \times 10^{5} \mathrm{lb} / \mathrm{h}\right)$ for the RTO system using the simplified efficiency curve model. These large plant movements result from the plant perturbation determined by the expected profit experimental design criterion. Relatively small changes in steam loads $\left(\|\Delta \mathrm{F}\|<0.3 \times 10^{5} \mathrm{lb} / \mathrm{h}\right)$ are observed in the direct search method because of the restriction of the plant movement from the chosen step size.

Case study results for other disturbance scenarios at different frequencies were studied. The RTO system using the fundamental model tracked the changing optimum closely for the disturbances considered at different frequencies. The RTO system using the efficiency curve model experienced larger efficiency losses. The direct search method takes many steps to reach the optimum, resulting in significant loss in efficiency during tracking even for slow disturbances.

\section{Conclusions}

The performance of three optimization strategies with different modelling approaches have been investigated: 1) model-free direct search method, 2) model-based method using the simplified efficiency curve model, and 3) model-based method using the fundamental model. For the commonly encountered disturbances in demand, fuel composition and heat exchanger fouling, case study results showed that using a high fidelity fundamental model could track the changing optimum closely at different disturbance frequency without experimentation. The disturbances could be corrected by updating the heat transfer coefficients and fuel heating value. The model-based RTO system using a simplified efficiency model required plant experimentation to correct for the disturbances in fuel composition and heat exchanger fouling. There was a significant profit loss during experimentation and tracking which was not desirable for tracking fast disturbances. The model-free direct search method took a lot of steps to reach the optimum, and a significant loss in boiler efficiency was observed during transient.

The engineer must select the model fidelity, RTO approach, and sensors in a consistent manner. In the boiler case, the more complex fundamental model requires less expensive sensors. The simper, efficiency curve model requires less model development, but it requires a heating value sensor and periodic experiments for model updating. The direct search approach requires no fundamental modelling and simple real-time calculations, but it also requires the heating value sensor.

Based on the case study results, a fundamental model is always advantageous for this RTO application. However, the cost of model development is also a concern for practicing engineers, and the simplified efficiency curve model might be a good compromise in some cases. The direct search has poorer performance and high sensor cost, and it is not recommended.

\section{References}

Bozenhardt, H. (1986). Hyperplane: A Case History. Proc. Fifth Annual Control Engineering Conference.

Cutler, C. R. and Perry, R. T. (1983). Real-Time Optimization with Multivariable Control Is Required to Maximize Profits. Computers and Chemical Engineering, 7(5), 663-667.

Darby, M. L. and White, D. C. (1988). On-Line Optimization of Complex Processes. Chemical Engineering Progress, 51-59.

Dukelow, S. G. (1991). The Control of Boilers. $2^{\text {nd }}$ edition, Instrument Society of America.

Forbes, J. F. and Marlin, T. E. (1996). Design Cost: A Systematic Approach to Technology Selection for Model-Based Real-Time Optimization Systems. Computers and Chemical Engineering, 20(6/7), 717 734.

Green, D. and Al ai-Shaikh. (1980). Evaluating and Improving Efficiency in a Boiler Complex Through Computer Control. JACC. 
Marlin, T. E. and Hrymak, A. N. (1997). Real-Time Optimization of Continuous Processes. American Institute of Chemical Engineering Symposium Series - Fifth International Conference on Chemical Process Control, 93, 156-164.

Payne, F. W. (1985). Efficient Boiler Operations Sourcebook. The Fairmont Press, Inc.

Yip, W. S. (2002). Model Updating in Real-time Optimization. Ph.D. Dissertation. McMaster University, Hamilton ON, Canada.

Yip, W. S. and Marlin, T. E. (2001a). Multiple Data Sets for Model Updating in Real-Time Operations Optimization. Submitted to Computers and Chemical Engineering.

Yip, W. S. and Marlin, T. E. (2001b). Designing Plant Experiments for Real-Time Optimization Systems. $6^{\text {th }}$ IFAC Symposium on Dynamics and Control of Process Systems, Jejudo, Korea, June 4-6, 373-378.
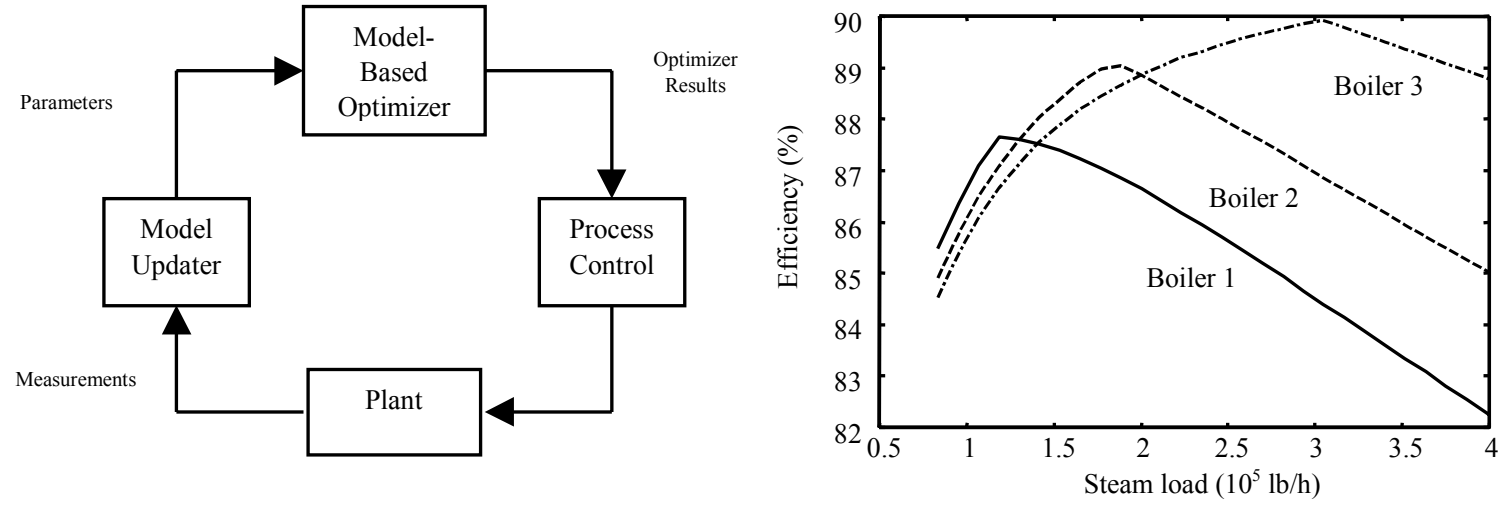

Figure 1 Closed-loop RTO system

Figure 2 Efficiency curve as a function of steam load

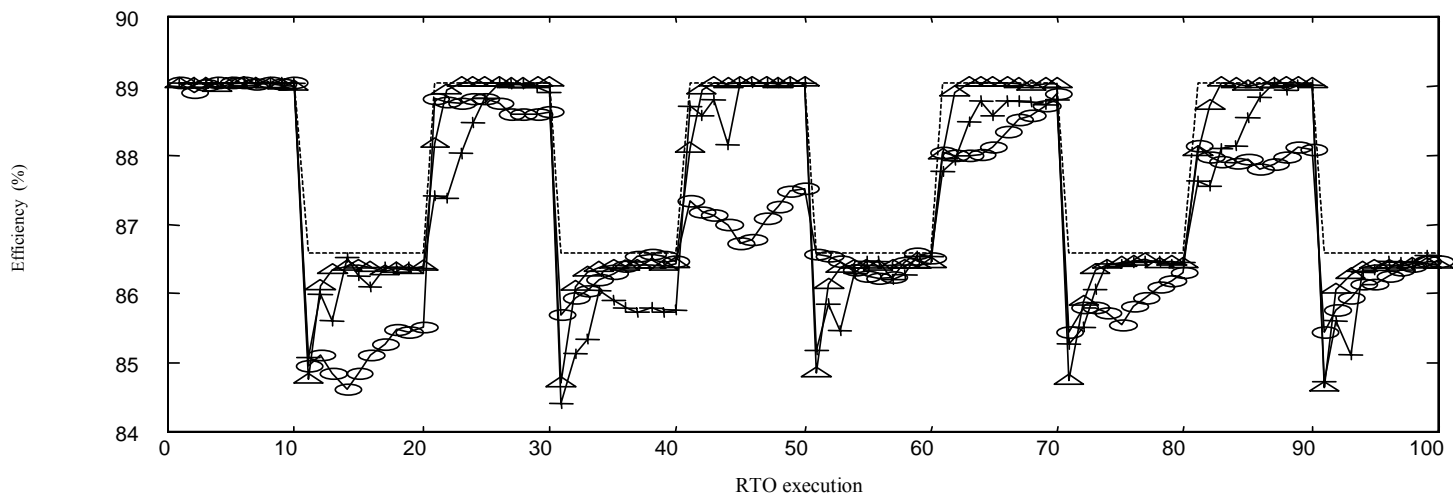

Figure 3 Boiler network efficiency attained for a fuel composition change (o : direct search, + : efficiency curve model, $\Delta$ : fundamental model, -- : true optimum)

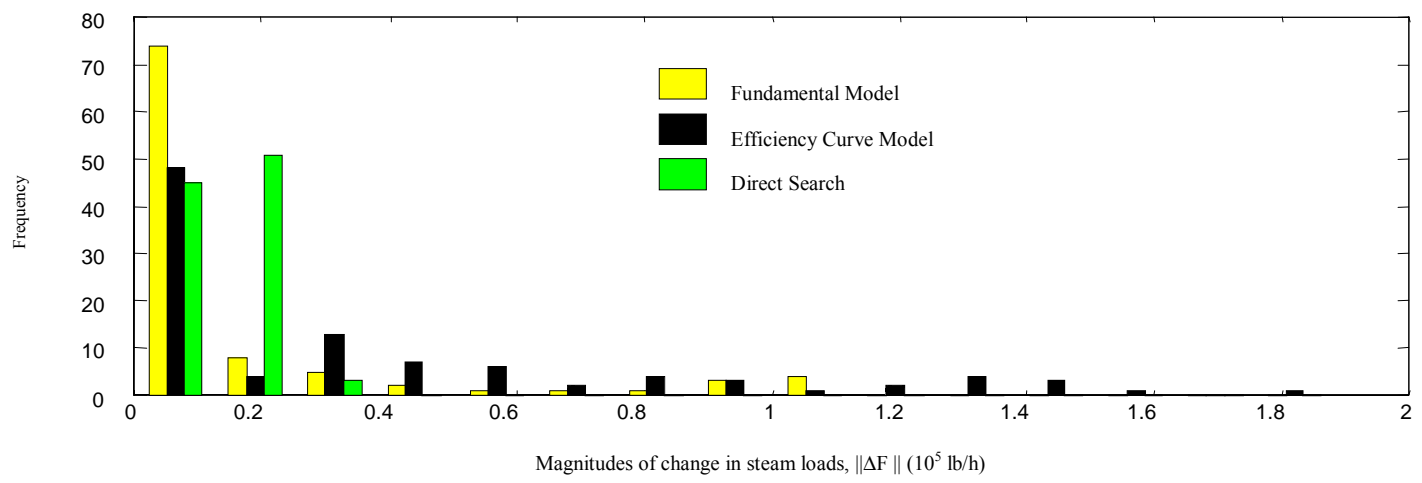

Figure 4 Distribution of the magnitude of change in steam loads for fuel composition change 